\title{
Estimates of Upper Bound for a Function Associated with Riemann-Liouville Fractional Integral via $h$-Convex Functions
}

\author{
Shan-He Wu $\mathbb{D D}^{1}$ and Muhammad Uzair Awan ${ }^{2}$ \\ ${ }^{1}$ Department of Mathematics, Longyan University, Longyan, 364012, China \\ ${ }^{2}$ Department of Mathematics, Government College University, Faisalabad, 313000, Pakistan \\ Correspondence should be addressed to Shan-He Wu; shanhewu@gmail.com
}

Received 2 February 2019; Accepted 7 April 2019; Published 2 May 2019

Academic Editor: Alberto Fiorenza

Copyright (c) 2019 Shan-He Wu and Muhammad Uzair Awan. This is an open access article distributed under the Creative Commons Attribution License, which permits unrestricted use, distribution, and reproduction in any medium, provided the original work is properly cited.

\begin{abstract}
A new identity involving Riemann-Liouville fractional integral is proposed. The result is then used to obtain some estimates of upper bound for a function associated with Riemann-Liouville fractional integral via $h$-convex functions. An application for establishing the inequalities related to special means is also considered.
\end{abstract}

\section{Introduction}

A set $K \subset \mathbb{R}$ is said to be convex, if

$$
(1-\mu) x+\mu y \in K, \quad \forall x, y \in K, \mu \in[0,1] .
$$

A function $f: K \longrightarrow \mathbb{R}$ is said to be convex, if

$$
\begin{aligned}
f((1-\mu) x+\mu y) \leq(1-\mu) f(x)+\mu f(y), & \\
& \forall x, y \in K, \mu \in[0,1] .
\end{aligned}
$$

In 1978, Breckner [1] introduced the concept of $s$-convex functions as a generalization of convex functions, as follows.

Definition 1 (see [1]). Let $s$ be a real number, $s \in(0,1]$. A function $f:[0, \infty) \longrightarrow[0, \infty)$ is said to be $s$-convex, if

$$
\begin{aligned}
f((1-\mu) x+\mu y) \leq(1-\mu)^{s} f(x)+\mu^{s} f(y) & \\
& \forall x, y \in[0, \infty), \mu \in[0,1] .
\end{aligned}
$$

In recent years several new extensions of classical convexity have been proposed in the literature. Varošanec [2] investigated a more generalized class of convex functions named $h$-convex function, as follows.
Definition 2 (see [2]). Let $h: J \longrightarrow \mathbb{R}$ be a nonnegative function. A function $f: I \longrightarrow \mathbb{R}$ is said to be $h$-convex, if

$$
\begin{array}{r}
f((1-\mu) x+\mu y) \leq h(1-\mu) f(x)+h(\mu) f(y), \\
\forall x, y \in I, \mu \in(0,1) .
\end{array}
$$

It has been observed that the class of $h$-convex functions unifies several other classes of convexity; for example, if we take $h(\mu)=\mu^{s}, h(\mu)=\mu^{-1}, h(\mu)=\mu^{-s}$, and $h(\mu)=1$, respectively, then we have the class of $s$-convex functions [1], the class of Q-functions [3], the class of $s$-Godunova-Levin type functions [4], and the class of $P$-functions [5]. For more details on convexity and its generalizations, see [6-9].

Convexity of a function plays a vital role in theory of inequalities, because many inequalities can easily be obtained using the functions having convexity properties. Hermite and Hadamard's result which is known as Hermite-Hadamard's inequality is one of the most fascinating results in the field of integral inequalities. This inequality provides a lower and an upper estimate for the integral average of any convex function defined on an interval. This famous result reads as follows.

Let $f:[a, b] \longrightarrow \mathbb{R}$ be a convex function, then

$$
f\left(\frac{a+b}{2}\right) \leq \frac{1}{b-a} \int_{a}^{b} f(x) \mathrm{d} x \leq \frac{f(a)+f(b)}{2} .
$$


Sarikaya et al. [10] gave a generalization of HermiteHadamard's inequality using the $h$-convexity of the function as follows.

Let $f:[a, b] \longrightarrow \mathbb{R}$ be $h$-convex function, then, for $h(1 / 2) \neq 0$, we have

$$
\begin{aligned}
\frac{1}{2 h(1 / 2)} f\left(\frac{a+b}{2}\right) & \leq \frac{1}{b-a} \int_{a}^{b} f(x) \mathrm{d} x \\
& \leq[f(a)+f(b)] \int_{0}^{1} h(\mu) \mathrm{d} \mu .
\end{aligned}
$$

Although the fractional calculus has a long history, it plays significant role in different fields of pure and applied mathematics [11]. Up to now, the study of the fractional calculus is still very active. Sarikaya et al. [12] used the concepts of Riemann-Liouville integrals to obtain the fractional version of Hermite-Hadamard's inequality. In fact, there are numerous new inequalities that have been obtained using the techniques of fractional calculus. For more details, see [1215].

In this paper, we present a new integral identity for differentiable functions involving fractional integrals. Then using this auxiliary result we establish our main results that are the estimates of upper bound for a function associated with Riemann-Liouville fractional integral via $h$-convex functions. At the end of the paper, we give an application of the obtained results to the special means.

We begin with recalling the definition of RiemannLiouville fractional integrals, as follows.

Definition 3 (see [11]). Let $f \in L_{1}[a, b]$. The RiemannLiouville integrals $J_{a^{+}}^{\alpha} f$ and $J_{b^{-}}^{\alpha} f$ of order $\alpha>0$ with $a \geq 0$ are defined by

$$
J_{a^{+}}^{\alpha} f(x)=\frac{1}{\Gamma(\alpha)} \int_{a}^{x}(x-\mu)^{\alpha-1} f(\mu) \mathrm{d} \mu, \quad x>a,
$$

and

$$
J_{b^{-}}^{\alpha} f(x)=\frac{1}{\Gamma(\alpha)} \int_{x}^{b}(\mu-x)^{\alpha-1} f(\mu) \mathrm{d} \mu, \quad x<b,
$$

where

$$
\Gamma(\alpha)=\int_{0}^{\infty} \mu^{\alpha-1} e^{-\mu} d \mu
$$

is the Gamma function.

The integral form of the hypergeometric function is

$$
\begin{aligned}
& { }_{2} F_{1}(x, y ; c ; z) \\
& =\frac{1}{\mathrm{~B}(y, c-y)} \int_{0}^{1} \mu^{y-1}(1-\mu)^{c-y-1}(1-z \mu)^{-x} \mathrm{~d} \mu, \\
& \quad \text { for }|z|\langle 1, c\rangle y\rangle 0,
\end{aligned}
$$

where $B(x, y)=\int_{0}^{1} \mu^{x-1}(1-\mu)^{y-1} \mathrm{~d} \mu$ is the Beta function.

\section{Main Results}

In this section, we consider the estimates of upper bound for the function below, which is associated with RiemannLiouville fractional integral. Consider the following:

$$
\begin{aligned}
& \Psi(a, b ; n ; \alpha)(f) \\
& \quad=\frac{(n+1)^{\alpha-1} \Gamma(\alpha+1)}{(b-a)^{\alpha}}\left[J_{((n a+b) /(n+1))^{-}}^{\alpha} f(a)\right. \\
& \left.\quad+J_{((a+n b) /(n+1))^{+}}^{\alpha} f(b)\right]-\frac{1}{n+1}\left[f\left(\frac{n a+b}{n+1}\right)\right. \\
& \left.\quad+f\left(\frac{a+n b}{n+1}\right)\right] .
\end{aligned}
$$

In order to establish the estimates of upper bound for $\Psi(a, b ; n ; \alpha)(f)$, we first prove an auxiliary result which plays an important role in dealing with subsequent results.

Lemma 4. Let $f:[a, b] \longrightarrow \mathbb{R}$ be differentiable function on $(a, b)$ with $0<a<b$. If $f^{\prime} \in L[a, b], \alpha>0$, and $n \in \mathbb{N}$, then

$$
\begin{aligned}
& \Psi(a, b ; n ; \alpha)(f)=\frac{b-a}{(n+1)^{2}} \int_{0}^{1}(1-\mu)^{\alpha} \\
& \cdot\left[-f^{\prime}\left(\frac{n+\mu}{n+1} a+\frac{1-\mu}{n+1} b\right)\right. \\
& \left.+f^{\prime}\left(\frac{1-\mu}{n+1} a+\frac{n+\mu}{n+1} b\right)\right] \mathrm{d} \mu \\
& \quad=\frac{(n+1)^{\alpha-1} \Gamma(\alpha+1)}{(b-a)^{\alpha}}\left[J_{((n a+b) /(n+1))^{-}}^{\alpha} f(a)\right. \\
& \left.+J_{((a+n b) /(n+1))^{+}}^{\alpha} f(b)\right]-\frac{1}{n+1}\left[f\left(\frac{n a+b}{n+1}\right)\right. \\
& \left.+f\left(\frac{a+n b}{n+1}\right)\right] .
\end{aligned}
$$

Proof. Let

$$
\begin{aligned}
I= & \int_{0}^{1}(1-\mu)^{\alpha}\left[-f^{\prime}\left(\frac{n+\mu}{n+1} a+\frac{1-\mu}{n+1} b\right)\right. \\
& \left.+f^{\prime}\left(\frac{1-\mu}{n+1} a+\frac{n+\mu}{n+1} b\right)\right] \mathrm{d} \mu=-\int_{0}^{1}(1 \\
& -\mu)^{\alpha} f^{\prime}\left(\frac{n+\mu}{n+1} a+\frac{1-\mu}{n+1} b\right) \mathrm{d} \mu+\int_{0}^{1}(1-\mu)^{\alpha} \\
& \cdot f^{\prime}\left(\frac{1-\mu}{n+1} a+\frac{n+\mu}{n+1} b\right) \mathrm{d} \mu=-I_{1}+I_{2} .
\end{aligned}
$$


Integrating $I_{1}$ gives

$$
\begin{aligned}
I_{1} & =\int_{0}^{1}(1-\mu)^{\alpha} f^{\prime}\left(\frac{n+\mu}{n+1} a+\frac{1-\mu}{n+1} b\right) \mathrm{d} \mu=\frac{n+1}{b-a} \\
& \cdot f\left(\frac{n}{n+1} a+\frac{1}{n+1} b\right)-\frac{(n+1) \alpha}{b-a} \\
& \cdot \int_{a}^{(n /(n+1)) a+(1 /(n+1)) b}(n+1)^{\alpha-1}\left(\frac{u-a}{b-a}\right)^{\alpha-1} f(u) \\
& \cdot \frac{n+1}{b-a} d u=\frac{n+1}{b-a} f\left(\frac{n}{n+1} a+\frac{1}{n+1} b\right) \\
& -\frac{(n+1)^{\alpha+1} \Gamma(\alpha+1)}{(b-a)^{\alpha+1}} J_{((n /(n+1)) a+(1 /(n+1)) b)^{-}}^{\alpha} f(a) .
\end{aligned}
$$

Similarly integrating $I_{2}$, one has

$$
\begin{aligned}
I_{2}= & \int_{0}^{1}(1-\mu)^{\alpha} f^{\prime}\left(\frac{1-\mu}{n+1} a+\frac{n+\mu}{n+1} b\right) \mathrm{d} \mu=-\frac{n+1}{b-a} \\
& \cdot f\left(\frac{1}{n+1} a+\frac{n}{n+1} b\right)+\frac{(n+1) \alpha}{b-a} \\
& \cdot \int_{(1 /(n+1)) a+(n /(n+1)) b}^{b}(n+1)^{\alpha-1}\left(\frac{b-v}{b-a}\right)^{\alpha-1} f(v) \\
& \cdot \frac{n+1}{b-a} d v=-\frac{n+1}{b-a} f\left(\frac{1}{n+1} a+\frac{n}{n+1} b\right) \\
& +\frac{(n+1)^{\alpha+1} \Gamma(\alpha+1)}{(b-a)^{\alpha+1}} J_{((1 /(n+1)) a+(n /(n+1)) b)^{+}}^{\alpha} f(b) .
\end{aligned}
$$

Using (14) and (15) in (13) leads to the identity described in Lemma 4.

Based on Lemma 4, we are now in a position to establish our main results.

Theorem 5. Let $f:[a, b] \longrightarrow \mathbb{R}$ be differentiable function on $(a, b)$ with $0<a<b$. If $f^{\prime} \in L[a, b], \alpha>0$, and $n \in \mathbb{N}$ and $\left|f^{\prime}\right|$ is h-convex function, then

$$
\begin{aligned}
& |\Psi(a, b ; n ; \alpha)(f)| \\
& \quad \leq \frac{b-a}{(n+1)^{2}}\left[\left|f^{\prime}(a)\right|+\left|f^{\prime}(b)\right|\right] \psi(n ; \alpha ; \mu),
\end{aligned}
$$

where

$$
\begin{aligned}
& \psi(n ; \alpha ; \mu) \\
& \quad=\int_{0}^{1}(1-\mu)^{\alpha}\left[h\left(\frac{n+\mu}{n+1}\right)+h\left(\frac{1-\mu}{n+1}\right)\right] \mathrm{d} \mu .
\end{aligned}
$$

Proof. Using Lemma 4 and the fact that $\left|f^{\prime}\right|$ is $h$-convex function, we have

$$
\begin{aligned}
& |\Psi(a, b ; n ; \alpha)(f)|=\mid \frac{b-a}{(n+1)^{2}} \int_{0}^{1}(1-\mu)^{\alpha} \\
& .\left[-f^{\prime}\left(\frac{n+\mu}{n+1} a+\frac{1-\mu}{n+1} b\right)\right. \\
& \left.+f^{\prime}\left(\frac{1-\mu}{n+1} a+\frac{n+\mu}{n+1} b\right)\right] \mathrm{d} \mu|\leq| \frac{b-a}{(n+1)^{2}} \\
& . \int_{0}^{1}(1-\mu)^{\alpha} f^{\prime}\left(\frac{n+\mu}{n+1} a+\frac{1-\mu}{n+1} b\right) \mathrm{d} \mu \mid \\
& +\left|\frac{b-a}{(n+1)^{2}} \int_{0}^{1}(1-\mu)^{\alpha} f^{\prime}\left(\frac{1-\mu}{n+1} a+\frac{n+\mu}{n+1} b\right) \mathrm{d} \mu\right| \\
& \leq \frac{b-a}{(n+1)^{2}} \int_{0}^{1}(1-\mu)^{\alpha}\left|f^{\prime}\left(\frac{n+\mu}{n+1} a+\frac{1-\mu}{n+1} b\right)\right| \mathrm{d} \mu \\
& +\frac{b-a}{(n+1)^{2}} \int_{0}^{1}(1-\mu)^{\alpha}\left|f^{\prime}\left(\frac{1-\mu}{n+1} a+\frac{n+\mu}{n+1} b\right)\right| \mathrm{d} \mu \\
& \quad \leq \frac{b-a}{(n+1)^{2}}\left[\int _ { 0 } ^ { 1 } ( 1 - \mu ) ^ { \alpha } \left[h\left(\frac{n+\mu}{n+1}\right)\right.\right.
\end{aligned}
$$

This completes the proof of Theorem 5 .

We now discuss some special cases which can be deduced directly from Theorem 5 .

(I) Putting $h(\mu)=\mu$ in Theorem 5, we have the following.

Corollary 6. Let $f:[a, b] \longrightarrow \mathbb{R}$ be differentiable function on $(a, b)$ with $0<a<b$. If $f^{\prime} \in L[a, b], \alpha>0$, and $n \in \mathbb{N}$ and $\left|f^{\prime}\right|$ is convex function, then

$$
\begin{aligned}
& |\Psi(a, b ; n ; \alpha)(f)| \\
& \quad \leq \frac{b-a}{(n+1)^{2}(\alpha+1)}\left[\left|f^{\prime}(a)\right|+\left|f^{\prime}(b)\right|\right] .
\end{aligned}
$$

(II) Putting $h(\mu)=\mu^{s}(0<s \leq 1)$ in Theorem 5, we have the following.

Corollary 7. Let $f:[a, b] \longrightarrow \mathbb{R}$ be differentiable function on $(a, b)$ with $0<a<b$. If $f^{\prime} \in L[a, b], \alpha>0$, and $n \in \mathbb{N}$ and $\left|f^{\prime}\right|$ is s-convex function, then

$$
\begin{aligned}
& |\Psi(a, b ; n ; \alpha)(f)| \\
& \quad \leq \frac{b-a}{(n+1)^{s+2}}\left[\frac{n_{2}^{s} F_{1}[-s, 1,2+\alpha,-1 / n]}{\alpha+1}\right. \\
& \left.\quad+\frac{1}{s+\alpha+1}\right]\left[\left|f^{\prime}(a)\right|+\left|f^{\prime}(b)\right|\right] .
\end{aligned}
$$

(III) Taking $h(\mu)=1$ in Theorem 5, we have the following. 
Corollary 8. Let $f:[a, b] \rightarrow \mathbb{R}$ be differentiable function on $(a, b)$ with $0<a<b$. If $f^{\prime} \in L[a, b], \alpha>0$, and $n \in \mathbb{N}$ and $\left|f^{\prime}\right|$ is P-function, then

$$
\begin{aligned}
& |\Psi(a, b ; n ; \alpha)(f)| \\
& \quad \leq \frac{2(b-a)}{(n+1)^{2}(\alpha+1)}\left[\left|f^{\prime}(a)\right|+\left|f^{\prime}(b)\right|\right] .
\end{aligned}
$$

(IV) Taking $h(\mu)=\mu^{-s}(0<s \leq 1)$ in Theorem 5, we have the following.

Corollary 9. Let $f:[a, b] \longrightarrow \mathbb{R}$ be differentiable function on $(a, b)$ with $0<a<b$. If $f^{\prime} \in L[a, b], \alpha>0$, and $n \in \mathbb{N}$ and $\left|f^{\prime}\right|$ is s-Godunova-Levin type function, then

$$
\begin{aligned}
& |\Psi(a, b ; n ; \alpha)(f)| \\
& \quad \leq \frac{b-a}{(n+1)^{2-s}}\left[\frac{n_{2}^{-s} F_{1}[s, 1,2+\alpha,-1 / n]}{\alpha+1}\right. \\
& \left.\quad+\frac{1}{\alpha-s+1}\right]\left[\left|f^{\prime}(a)\right|+\left|f^{\prime}(b)\right|\right] .
\end{aligned}
$$

Theorem 10. Let $f:[a, b] \longrightarrow \mathbb{R}$ be differentiable function on $(a, b)$ with $0<a<b, f^{\prime} \in L[a, b], \alpha>0$, and $n \in \mathbb{N}$, and let $\left|f^{\prime}\right|^{q}$ be h-convex function, $1 / p+1 / q=1, p, q>0$. Then

$$
\begin{aligned}
& |\Psi(a, b ; n ; \alpha)(f)| \leq \frac{b-a}{(n+1)^{2}}\left(\frac{1}{\alpha p+1}\right)^{1 / p} \\
& .\left[\left(\left|f^{\prime}(a)\right|^{q} \int_{0}^{1} h\left(\frac{n+\mu}{n+1}\right) \mathrm{d} \mu\right.\right. \\
& \left.+\left|f^{\prime}(b)\right|^{q} \int_{0}^{1} h\left(\frac{1-\mu}{n+1}\right) \mathrm{d} \mu\right)^{1 / q} \\
& +\left(\left|f^{\prime}(a)\right|^{q} \int_{0}^{1} h\left(\frac{1-\mu}{n+1}\right) \mathrm{d} \mu\right. \\
& \left.\left.+\left|f^{\prime}(b)\right|^{q} \int_{0}^{1} h\left(\frac{n+\mu}{n+1}\right) \mathrm{d} \mu\right)^{1 / q}\right] .
\end{aligned}
$$

Proof. Using Lemma 4, the Hölder inequality and the fact that $\left|f^{\prime}\right|^{q}$ is $h$-convex function, we have

$$
\begin{aligned}
& |\Psi(a, b ; n ; \alpha)(f)|=\mid \frac{b-a}{(n+1)^{2}} \int_{0}^{1}(1-\mu)^{\alpha} \\
& \cdot\left[-f^{\prime}\left(\frac{n+\mu}{n+1} a+\frac{1-\mu}{n+1} b\right)\right. \\
& \left.+f^{\prime}\left(\frac{1-\mu}{n+1} a+\frac{n+\mu}{n+1} b\right)\right] \mathrm{d} \mu|\leq| \frac{b-a}{(n+1)^{2}} \\
& \cdot \int_{0}^{1}(1-\mu)^{\alpha} f^{\prime}\left(\frac{n+\mu}{n+1} a+\frac{1-\mu}{n+1} b\right) \mathrm{d} \mu \mid \\
& +\left|\frac{b-a}{(n+1)^{2}} \int_{0}^{1}(1-\mu)^{\alpha} f^{\prime}\left(\frac{1-\mu}{n+1} a+\frac{n+\mu}{n+1} b\right) \mathrm{d} \mu\right|
\end{aligned}
$$

$$
\begin{aligned}
& \leq \frac{b-a}{(n+1)^{2}}\left(\int_{0}^{1}(1-\mu)^{\alpha p} \mathrm{~d} \mu\right)^{1 / p} \\
& \cdot\left(\int_{0}^{1}\left|f^{\prime}\left(\frac{n+\mu}{n+1} a+\frac{1-\mu}{n+1} b\right)\right|^{q} d t\right)^{1 / q} \\
& +\frac{b-a}{(n+1)^{2}}\left(\int_{0}^{1}(1-\mu)^{\alpha p} \mathrm{~d} \mu\right)^{1 / p} \\
& \cdot\left(\int_{0}^{1}\left|f^{\prime}\left(\frac{1-\mu}{n+1} a+\frac{n+\mu}{n+1} b\right)\right|^{q} d t\right)^{1 / q} \\
& \leq \frac{b-a}{(n+1)^{2}}\left(\frac{1}{\alpha p+1}\right)^{1 / p}\left[\left(\left|f^{\prime}(a)\right|^{q}\right.\right. \\
& \cdot \int_{0}^{1} h\left(\frac{n+\mu}{n+1}\right) \mathrm{d} \mu+\left|f^{\prime}(b)\right|^{q} \\
& \left.\cdot \int_{0}^{1} h\left(\frac{1-\mu}{n+1}\right) \mathrm{d} \mu\right)^{1 / q}+\left(\left|f^{\prime}(a)\right|^{q}\right. \\
& \cdot \int_{0}^{1} h\left(\frac{1-\mu}{n+1}\right) \mathrm{d} \mu+\left|f^{\prime}(b)\right|^{q} \\
& \left.\left.\cdot \int_{0}^{1} h\left(\frac{n+\mu}{n+1}\right) \mathrm{d} \mu\right)^{1 / q}\right] \text {. }
\end{aligned}
$$

The proof of Theorem 10 is complete.

We give now four corollaries that follow from the special cases of Theorem 10 .

(I) Choosing $h(\mu)=\mu$ in Theorem 10, we have the following.

Corollary 11. Let $f:[a, b] \longrightarrow \mathbb{R}$ be differentiable function on $(a, b)$ with $0<a<b, f^{\prime} \in L[a, b], \alpha>0$, and $n \in \mathbb{N}$, and let $\left|f^{\prime}\right|^{q}$ be convex function, $1 / p+1 / q=1, p, q>0$. Then

$$
\begin{aligned}
& |\Psi(a, b ; n ; \alpha)(f)| \\
& \leq \frac{b-a}{(n+1)^{2+1 / q}(\alpha p+1)^{1 / p}}\left[\left(\frac{2 n+1}{2}\left|f^{\prime}(a)\right|^{q}\right.\right. \\
& \left.\quad+\frac{1}{2}\left|f^{\prime}(b)\right|^{q}\right)^{1 / q}+\left(\frac{1}{2}\left|f^{\prime}(a)\right|^{q}\right. \\
& \left.\left.+\frac{2 n+1}{2}\left|f^{\prime}(b)\right|\right)^{1 / q}\right] .
\end{aligned}
$$

(II) Choosing $h(\mu)=\mu^{s}(0<s \leq 1)$ in Theorem 10, we have the following.

Corollary 12. Let $f:[a, b] \longrightarrow \mathbb{R}$ be differentiable function on $(a, b)$ with $0<a<b, f^{\prime} \in L[a, b], \alpha>0$, and $n \in \mathbb{N}$, and 
let $\left|f^{\prime}\right|^{q}$ be s-convex function, $1 / p+1 / q=1, p, q>0$. Then

$$
\begin{aligned}
& |\Psi(a, b ; n ; \alpha)(f)| \\
& \leq \frac{b-a}{(n+1)^{2+s / q}(\alpha p+1)^{1 / p}}\left[\left(\left(\frac{(n+1)^{s+1}-n^{s+1}}{s+1}\right)\right.\right. \\
& \left.\cdot\left|f^{\prime}(a)\right|^{q}+\frac{1}{s+1}\left|f^{\prime}(b)\right|^{q}\right)^{1 / q}+\left(\frac{1}{s+1}\left|f^{\prime}(a)\right|^{q}\right. \\
& \left.\left.\quad+\left(\frac{(n+1)^{s+1}-n^{s+1}}{s+1}\right)\left|f^{\prime}(b)\right|^{q}\right)^{1 / q}\right] .
\end{aligned}
$$

(III) Putting $h(\mu)=1$ in Theorem 10, we have the following.

Corollary 13. Let $f:[a, b] \longrightarrow \mathbb{R}$ be differentiable function on $(a, b)$ with $0<a<b, f^{\prime} \in L[a, b], \alpha>0$, and $n \in \mathbb{N}$, and let $\left|f^{\prime}\right|^{q}$ be P-function, $1 / p+1 / q=1, p, q>0$. Then

$$
\begin{aligned}
& |\Psi(a, b ; n ; \alpha)(f)| \\
& \quad \leq \frac{2(b-a)}{(n+1)^{2}(\alpha p+1)^{1 / p}}\left(\left|f^{\prime}(a)\right|^{q}+\left|f^{\prime}(b)\right|^{q}\right)^{1 / q} .
\end{aligned}
$$

(IV) Putting $h(\mu)=\mu^{-s}(0<s \leq 1)$ in Theorem 10, we have the following.

Corollary 14. Let $f:[a, b] \longrightarrow \mathbb{R}$ be differentiable function on $(a, b)$ with $0<a<b, f^{\prime} \in L[a, b], \alpha>0$, and $n \in \mathbb{N}$, and let $\left|f^{\prime}\right|^{q}$ be s-Godunova-Levin type function, $1 / p+1 / q=$ $1, p, q>0$. Then

$$
\begin{aligned}
& |\Psi(a, b ; n ; \alpha)(f)| \\
& \leq \frac{b-a}{(n+1)^{2-s / q}(\alpha p+1)^{1 / p}}\left[\left(\left(\frac{(n+1)^{1-s}-n^{1-s}}{1-s}\right)\right.\right. \\
& \left.\cdot\left|f^{\prime}(a)\right|^{q}+\frac{1}{1-s}\left|f^{\prime}(b)\right|^{q}\right)^{1 / q}+\left(\frac{1}{1-s}\left|f^{\prime}(a)\right|^{q}\right. \\
& \left.\left.\quad+\left(\frac{(n+1)^{1-s}-n^{1-s}}{1-s}\right)\left|f^{\prime}(b)\right|^{q}\right)^{1 / q}\right] .
\end{aligned}
$$

\section{Application to Special Means}

In this section, we give an application of the obtained results to special means.

Definition 15 (see [16]). Recall the following definitions.

(1) For arbitrary positive numbers $a, b(a \neq b)$,

$$
L(a, b)=\frac{b-a}{\log b-\log a}
$$

is called the logarithmic mean.
(2) For arbitrary real numbers $a, b$,

$$
A(a, b)=\frac{a+b}{2}
$$

is called the arithmetic mean.

(3) The extended logarithmic mean of two positive numbers $a, b(a \neq b)$ is defined by

$$
L_{p}(a, b)= \begin{cases}{\left[\frac{b^{p+1}-a^{p+1}}{(p+1)(b-a)}\right]^{1 / p},} & p \neq-1,0, \\ \frac{b-a}{\log b-\log a}, & p=-1, \\ \frac{1}{e}\left(\frac{b^{b}}{a^{a}}\right)^{1 /(b-a)}, & p=0 .\end{cases}
$$

We focus on the estimation of upper bound for the difference between logarithmic mean and arithmetic mean; we shall establish two inequalities related to these means.

Proposition 16. If $0<a<b, 0<s \leq 1$, then

$$
\left|L_{s}^{s}(a, b)-A^{s}(a, b)\right| \leq \vartheta(s)\left(a^{s-1}+b^{s-1}\right)
$$

where

$$
\vartheta(s)=\frac{s(b-a)}{2^{s+2}}\left[\frac{{ }_{2} F_{1}[-s, 1,3,-1]}{2}+\frac{1}{s+2}\right] .
$$

Proof. We start by verifying that $\varphi(x)=s x^{s-1}(0<s \leq 1)$ is $s$-convex on $(0, \infty)$.

In view of

$$
\varphi^{\prime \prime}(x)=s(s-1)(s-2) x^{s-3} \geq 0 \text { for } 0<s \leq 1,
$$

which implies that $\varphi(x)$ is convex on $(0, \infty)$, thus for all $u, v \in$ $(0, \infty), t \in[0,1]$, and $0<s \leq 1$ we have

$$
\begin{aligned}
\varphi((1-t) u+t v) & =s((1-t) u+t v)^{s-1} \\
& \leq(1-t) s u^{s-1}+t s v^{s-1} \\
& \leq(1-t)^{s} s u^{s-1}+t^{s} s v^{s-1} \\
& =(1-t)^{s} \varphi(u)+t^{s} \varphi(v)
\end{aligned}
$$

Hence, $\varphi(x)=s x^{s-1}(0<s \leq 1)$ is $s$-convex on $(0, \infty)$.

Now, putting $f(x)=x^{s}, s \in(0,1), \alpha=1, n=1$ in Corollary 7, we obtain

$$
\begin{aligned}
\mid \Psi & (a, b ; n ; \alpha)(f) \mid \\
& =\left|\frac{1}{b-a}\left(\frac{b^{s+1}-a^{s+1}}{s+1}\right)-\left(\frac{a+b}{2}\right)^{s}\right| \\
& =\left|L_{s}^{s}(a, b)-A^{s}(a, b)\right|
\end{aligned}
$$


and

$$
\begin{aligned}
& \frac{b-a}{(n+1)^{s+2}}\left[\frac{n_{2}^{s} F_{1}[-s, 1,2+\alpha,-1 / n]}{\alpha+1}+\frac{1}{s+\alpha+1}\right] \\
& \cdot\left[\left|f^{\prime}(a)\right|+\left|f^{\prime}(b)\right|\right] \\
& =\frac{s(b-a)}{2^{s+2}}\left[\frac{{ }_{2} F_{1}[-s, 1,3,-1]}{2}+\frac{1}{s+2}\right]\left(a^{s-1}\right. \\
& \left.+b^{s-1}\right) .
\end{aligned}
$$

Note that the function $\left|f^{\prime}(x)\right|=s x^{s-1}(0<s \leq 1)$ is $s$-convex on $(0, \infty)$. Hence, the inequality (32) follows straightway from the inequality given in Corollary 7.

Proposition 17. If $0<a<b, 1 / p+1 / q=1, p>0, q>0$, and $0<s \leq 1$, then

$$
\begin{aligned}
& \left|L_{s}^{s}(a, b)-A^{s}(a, b)\right| \\
& \quad \leq \frac{s(b-a)}{2^{2+s / q}(p+1)^{1 / p}(s+1)^{1 / q}}\left(\eta_{1}^{1 / q}+\eta_{2}^{1 / q}\right),
\end{aligned}
$$

where

$$
\begin{aligned}
& \eta_{1}=\left(2^{s+1}-1\right) a^{q(s-1)}+b^{q(s-1)}, \\
& \eta_{2}=a^{q(s-1)}+\left(2^{s+1}-1\right) b^{q(s-1)} .
\end{aligned}
$$

Proof. Taking $f(x)=x^{s}(0<s \leq 1), \alpha=1, n=1$ in Corollary 12, we obtain

$$
\begin{aligned}
& |\Psi(a, b ; n ; \alpha)(f)| \\
& \quad=\left|\frac{1}{b-a}\left(\frac{b^{s+1}-a^{s+1}}{s+1}\right)-\left(\frac{a+b}{2}\right)^{s}\right| \\
& =\left|L_{s}^{s}(a, b)-A^{s}(a, b)\right|
\end{aligned}
$$

and

$$
\begin{aligned}
& \frac{b-a}{(n+1)^{2+s / q}(\alpha p+1)^{1 / p}}\left[\left(\frac{(n+1)^{s+1}-n^{s+1}}{s+1}\right)\right. \\
& \left.\cdot\left|f^{\prime}(a)\right|^{q}+\frac{1}{s+1}\left|f^{\prime}(b)\right|^{q}\right)^{1 / q}+\left(\frac{1}{s+1}\left|f^{\prime}(a)\right|^{q}\right. \\
& \left.\left.+\left(\frac{(n+1)^{s+1}-n^{s+1}}{s+1}\right)\left|f^{\prime}(b)\right|^{q}\right)^{1 / q}\right] \\
& =\frac{s(b-a)}{2^{2+s / q}(p+1)^{1 / p}(s+1)^{1 / q}}\left[\left(\left(2^{s+1}-1\right) a^{q(s-1)}\right.\right. \\
& \left.\left.+b^{q(s-1)}\right)^{1 / q}+\left(a^{q(s-1)}+\left(2^{s+1}-1\right) b^{q(s-1)}\right)^{1 / q}\right] .
\end{aligned}
$$

It remains to prove that $\phi(x)=s^{q} x^{q(s-1)}(0<s \leq 1, q>$ $0)$ is $s$-convex on $(0, \infty)$.
In fact, one has

$$
\begin{aligned}
& \phi^{\prime \prime}(x)=s^{q} q(s-1)(q(s-1)-1) x^{q(s-1)-2} \geq 0 \\
& \qquad \text { for } 0<s \leq 1, q>0 .
\end{aligned}
$$

It follows that $\phi(x)$ is convex on $(0, \infty)$; thus for all $u, v \in$ $(0, \infty), t \in[0,1]$, and $0<s \leq 1$ we have

$$
\begin{aligned}
\phi((1-t) u+t v) & \leq(1-t) \phi(u)+t \phi(v) \\
& \leq(1-t)^{s} \varphi(u)+t^{s} \varphi(v),
\end{aligned}
$$

which implies that $\phi(x)=s^{q} x^{q(s-1)}(0<s \leq 1, q>0)$ is $s$-convex on $(0, \infty)$.

Now, utilizing the fact that $\left|f^{\prime}(x)\right|^{q}=s^{q} x^{q(s-1)}(0<s \leq$ $1, q>0)$ is $s$-convex on $(0, \infty)$, we can deduce the desired inequality (38) from Corollary 12.

\section{Data Availability}

The datasets used or analysed during the current study are available from the corresponding author upon reasonable request.

\section{Conflicts of Interest}

The authors declare that they have no competing interests.

\section{Authors' Contributions}

All authors read and approved the final manuscript.

\section{Acknowledgments}

The work of the first author was supported by the Natural Science Foundation of Fujian Province of China (Grant no. 2016J01023) and the Teaching Reform Project of Longyan University (Grant no. 2017JZ02).

\section{References}

[1] W. W. Breckner, "Stetigkeitsaussagen für eine Klasse verallgemeinerter konvexer funktionen in topologischen linearen Räumen," Publications de l'Institut Mathématique, vol. 23, pp. 13-20, 1978.

[2] S. Varošanec, "On h-convexity," Journal of Mathematical Analysis and Applications, vol. 326, no. 1, pp. 303-311, 2007.

[3] E. K. Godunova and V. I. Levin, "Inequalities for functions of a broad class that contains convex, monotone and some other forms of functions (Russian)," in Numerical Mathematics and Mathematical Physics (Russian), pp. 138-142, 166, Moskov. Gos. Ped. Inst., Moscow, Russia, 1985.

[4] S. S. Dragomir, "Inequalities of Hermite-Hadamard type for h-convex functions on linear spaces," Proyecciones Journal of Mathematics, vol. 34, no. 4, pp. 323-341, 2015.

[5] S. S. Dragomir, J. Pečarić, and L. E. Persson, "Some inequalities of Hadamard type," Soochow Journal of Mathematics, vol. 21, no. 3, pp. 335-341, 1995. 
[6] G. Cristescu and L. Lupşa, Non-Connected Convexities and Applications, vol. 68 of Applied Optimization, Kluwer Academic Publishers, Dordrecht, Netherlands, 2002.

[7] G. Cristescu, M. A. Noor, and M. U. Awan, "Bounds of the second degree cumulative frontier gaps of functions with generalized convexity," Carpathian Journal of Mathematics, vol. 31, no. 2, pp. 173-180, 2015.

[8] S. S. Dragomir and C. E. M. Pearce, Selected Topics on HermiteHadamard Inequalities and Applications, Victoria University, Australia, 2000.

[9] M. Tunç, "On some integral inequalities via $h$-convexity," Miskolc Mathematical Notes, vol. 14, no. 3, pp. 1041-1057, 2013.

[10] M. Z. Sarikaya, A. Saglam, and H. Yildirim, "On some Hadamard-type inequalities for $h$-convex functions," Journal of Mathematical Inequalities, vol. 2, no. 3, pp. 335-341, 2008.

[11] A. A. Kilbas, H. M. Srivastava, and J. J. Trujillo, Theory and Applications of Fractional Differential Equations, vol. 204 of North-Holland Mathematics Studies, Elsevier Science B.V., Amsterdam, Netherlands, 2006.

[12] M. Z. Sarikaya, E. Set, H. Yaldiz, and N. Başak, "Hermite-Hadamard's inequalities for fractional integrals and related fractional inequalities," Mathematical and Computer Modelling, vol. 57, no. 9-10, pp. 2403-2407, 2013.

[13] M. A. Noor and M. U. Awan, "Some integral inequalities for two kinds of convexities via fractional integrals," Transylvanian Journal of Mathematics and Mechanics, vol. 5, no. 2, pp. 129-136, 2013.

[14] M. A. Noor, G. Cristescu, and M. U. Awan, "Generalized fractional Hermite-Hadamard inequalities for twice differentiable s-convex functions," Filomat, vol. 29, no. 4, pp. 807-815, 2015.

[15] M. A. Noor, K. I. Noor, M. U. Awan, and S. Khan, "Fractional Hermite-Hadamard inequalities for some new classes of Godunova-Levin functions," Applied Mathematics \& Information Sciences, vol. 8, no. 6, pp. 2865-2872, 2014.

[16] C. P. Niculescu and L. E. Persson, Convex Functions and Their Applications, Springer-Verlag, New York, NY, USA, 2006. 


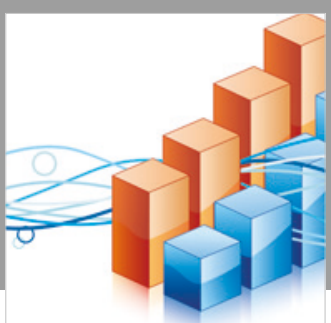

Advances in

Operations Research

\section{-n-m}
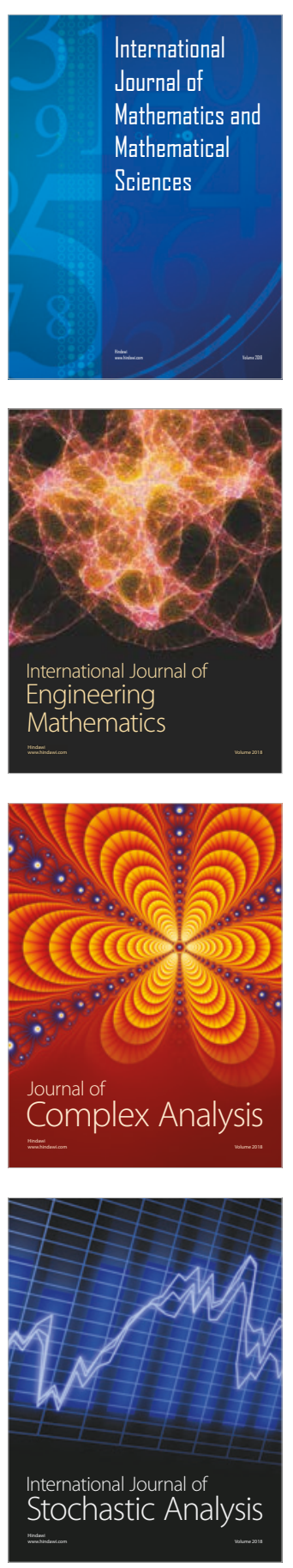
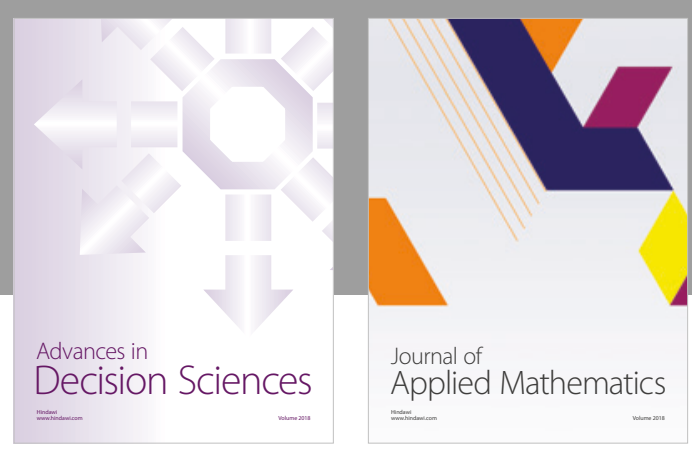

Journal of

Applied Mathematics
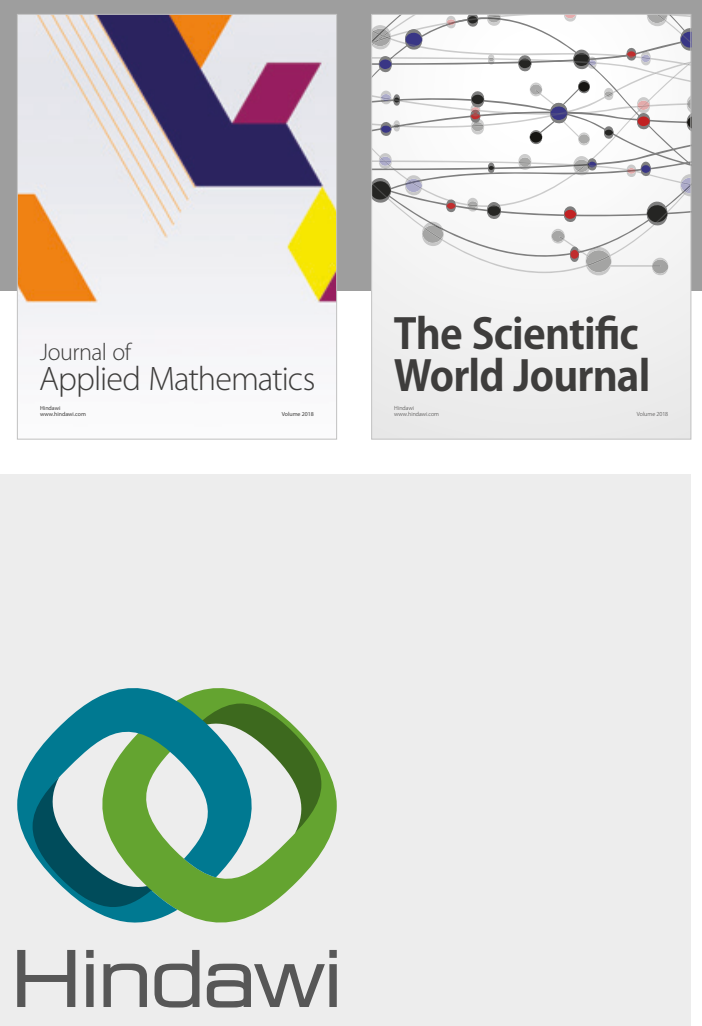

Submit your manuscripts at

www.hindawi.com

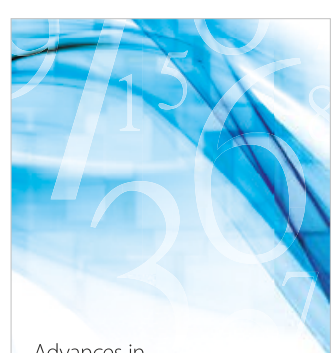

Advances in
Numerical Analysis
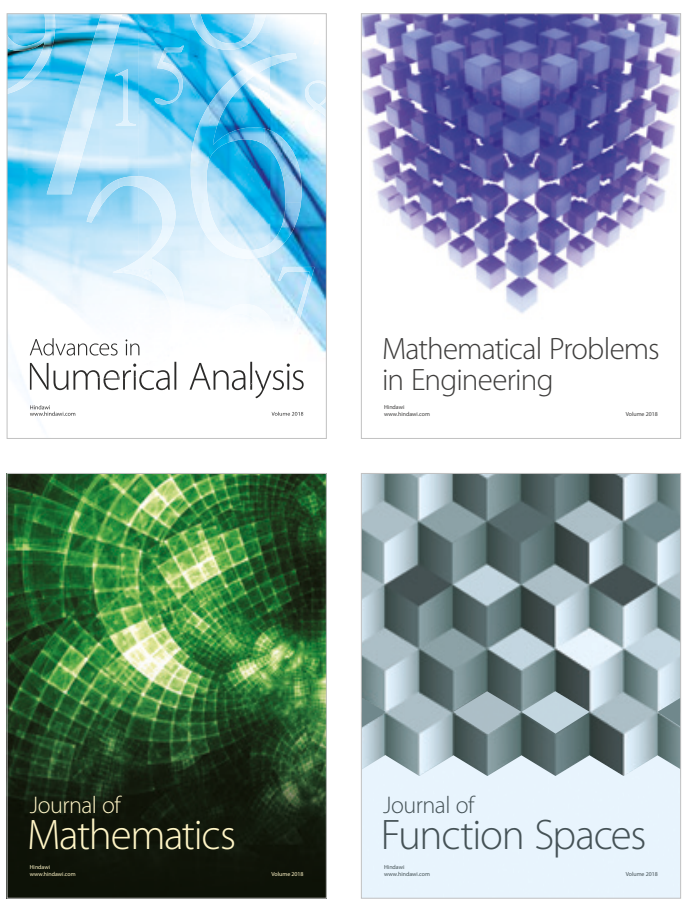

Mathematical Problems in Engineering

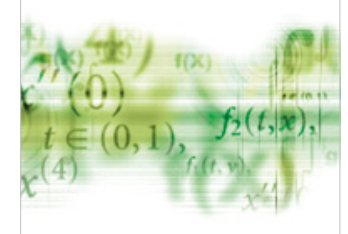

International Journal of

Differential Equations

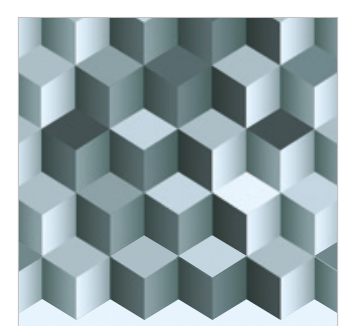

Journal of

Function Spaces

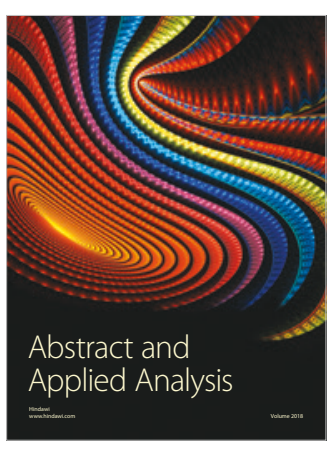

The Scientific

World Journal

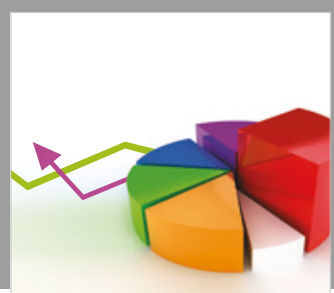

Journal of

Probability and Statistics
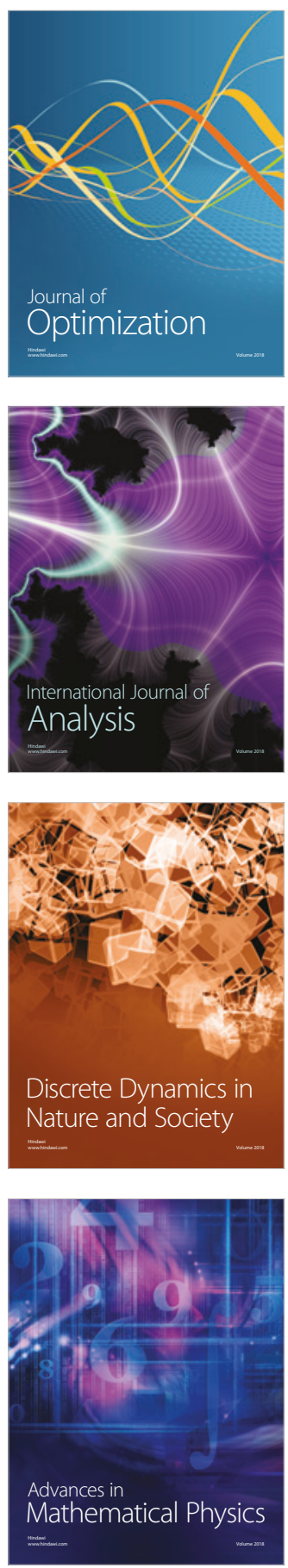